\title{
Construing domain knowledge via terminological understanding
}

\author{
Koen Kerremans, Rita Temmerman and Peter De Baer \\ Erasmushogeschool Brussel
}

For me it is a starting point in all thought about language that, whatever I say or do with words, my expression will never mean exactly the same thing to you that it does to me; and of course yours will never mean exactly the same thing to me that it does to you. It cannot: each act of expression is a gesture against a context.

(White 1991: 68)

This article will focus on the process of understanding terms, which is essential for translators to propose suitable translations and for terminologists to develop different types of terminological resources. We will discuss how a shift from meaning to understanding has changed our view with respect to the interaction between symbols (i.e. terms), thoughts and referents. Next, we will demonstrate how this view has been applied in termontography, a methodology set up to develop ontologically-underpinned terminological resources, based on the analysis of specialised texts. We will concentrate on the categorization framework, a concept used in termontography for structuring terminological information, and discuss how this framework is currently implemented in a didactic software tool, called CatTerm, which guides student translators to construct a knowledge model of a given domain.

\section{The process of understanding}

Discussions about the possible meanings of terms show resemblance to similar long-standing debates in lexical semantics about the possible meanings of words. Topics include, for example, the arbitrariness of the linguistic form, the question whether words possess meaning in isolation or acquire meaning only in sentences and the relation between meaning and reference. For details on the history of these ongoing discussions we refer to, e.g. Raskin (1986), Geeraerts (1986) and Rastier (1991).

In this article, we do not intend to enter into a theoretical discussion of meaning. Instead, we will deal with the process of understanding, which, for example, terminographers need to consider in order to develop different types of terminological resources. This process is also essential for translators who need to propose suitable translations. We will focus on the implications of this shift from meaning to understanding with respect to our own methodology in developing terminological resources. Special attention will also be devoted to how the methodology is used to teach student translators 
how to acquire domain knowledge in order to translate specialised texts (e.g. technical, scientific and legal texts). A bilingual corpus of specialised texts can help student translators acquire at least a basic insight into the domain at hand (e.g. Zanettin 1998, Bowker 1999, Wilkinson 2005), become familiar with the terms that are used in the domain (in both source and target languages) and become aware of semantic differences. We consider all this to be part of a domain knowledge acquisition process. From our own experience in teaching specialised translation courses, we have learned that this process is often underestimated by many of the aspiring translators. Most of their time goes to finding immediate solutions for translation problems. This is why we have developed a software tool (CatTerm) that guides student translators along different methodological steps in order to construct a knowledge model of a given domain.

Before we present the CatTerm software, we will show in Section 2. how our focus on understanding derives from the sociocognitive view in terminology (Temmerman 2000). In Section 3, we will reflect on possible implications of this view for terminographers and student translators. In Section 4, we will focus on termontography, a method set up to develop ontologically-underpinned terminological resources (see e.g. Kerremans et al. 2003), which has recently been implemented in the CatTerm software. This software tool will be presented in Section 5. Finally, in Section 6, we will present our conclusion.

\section{From meaning to understanding}

Ogden and Richards (1923) visualised the distinction between language symbols, thoughts (or references) and referents in a triangle, which is commonly known as the semiotic triangle, the triangle of reference or the triangle of meaning. The figure shows that thoughts are created in our minds to refer to referents (e.g. objects) in reality and that language symbols are used to symbolise these thoughts. Meaning is established on the basis of the relation between language symbols and thoughts referring to referents.

We add to this that, for example, Vygotsky (1986) perceived the relation between language symbols (words) and thoughts not as a thing but as a process, a continual movement back and forth from thoughts to words and from words to thoughts. In that process, the relation of thoughts to words undergoes constant changes, resulting from a view that thoughts are not merely expressed in words but come into existence through these words. This illustrates that the relation between words and thoughts is much more complex than what can be derived from the semiotic triangle. Words do not simply 'symbolise' but are also functional in the development process of thoughts. This implies that an investigation of the interaction of thoughts and words must begin with an investigation of the different phases a thought traverses before it is represented in words. 
In sociocognitive terminology, the shift from meaning to understanding changes our perception of the interaction between language symbols, thoughts and referents. The focus on understanding places the human subject or 'conceptualiser', who is able to understand and to create thoughts based on his understanding, at the centre of attention.

In cognitive linguistics it is believed that human subjects' thoughts about the world cannot be objectified, because many thoughts, representing their knowledge and understanding of the world, are embodied, that is, acquired via sensory perceptions (Chrisley and Ziemke 2002). A thought can never cover the aspects or features of a given referent all at once because of the many perspectives from which the referent can be approached or perceived. Human subjects will never share exactly the same thoughts about given referents because of the different ways in which these referents can be experienced. Focussing on understanding (instead of meaning) therefore allows us to emphasise the subjective nature of thoughts, which has important implications for the perception and treatment of terms, as we shall see later on in this section

Individual experience is considered to be situated, which means that the domain community's tradition conditions the embodied experience of each member. Consequently, thoughts are to some degree intersubjective as they emerge in a cultural group (e.g. country, educational institution, company, science lab) whose members share a more similar approach in the perception of referents and who may negotiate and renegotiate their cultural understanding across time and space. A similar approach or view does not mean that members of a cultural group share exactly the same thoughts about given referents. Cultural understanding is said to be distributed in the sense that it is not equally imprinted in the mind of each member (Sharifian 2003).

Thought or reference

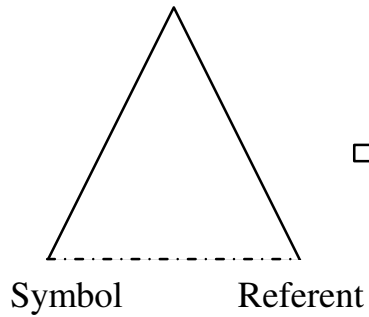

Thought or reference

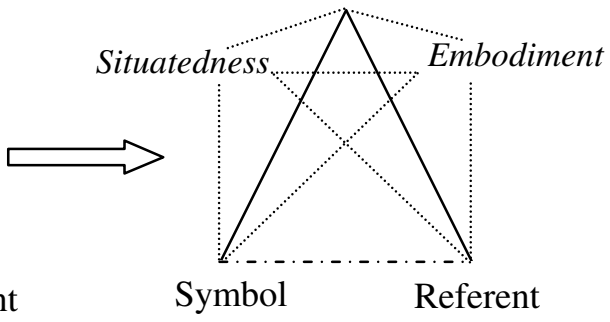

Figure 1: Extending the semiotic triangle

Adding the notions 'embodiment' and 'situatedness' to the semiotic triangle, as we have illustrated above, Figure 1 changes the perception of the relation between human understanding and language. Human understanding is believed to be prototypically structured, as has been put forward in cogni- 
tive linguistics (e.g. Fillmore 1985, Fillmore and Atkins 1992), which implies that a given referent can be categorised in different ways. From the point of view of cognitive linguistics, language is defined as a system for the categorisation of the world (e.g. Geeraerts 1997). It largely embodies individual and cultural categorisations and, consequently, reflects the prototypical structure of human understanding. In special language, for instance, the many possible ways to categorise a given referent may be one of the causes of denominative variation, whereby different terms denote the same referent even though they may symbolise different categorisations. This is the case for terms such as 'Southern blotting' and 'Southern transfer', which both refer to a method applied in molecular biology to check for the presence of a DNA sequence in a DNA sample. Temmerman (2000) argued that these denominative variants in fact result from differences in categorisation: 'Southern blotting' emphasises the result of the technique whereas 'Southern transfer' focuses on the process.

The idea of language reflecting situated understanding has been demonstrated on the basis of a diachronic study of the term 'splicing' in Temmerman (2008). The vantage point of this study has been to try to gain insight into understanding as it emerges from terms and descriptions in scientific publications (both in original scientific articles and in popularising literature). Terms reflect the dynamic, distributed and embodied nature of understanding. At some point, when a new insight occurs, when a new technique is developed or when a new phenomenon is discovered, there is understanding of some kind. Subject specialists can describe and name what they understand and create or use a term based on, for example, the intuitive meaning selection or extension of a lexeme. The selection or creation of a term is influenced not only by the (embodied) understanding of one individual. The naming activity always occurs in a particular environment and situation that influences the activity itself, for example, in a research laboratory (Temmerman 2008).

Understanding is not only diversified. It is also flexible as it derives from a continuously evolving process which relates present perceptions and knowledge via reflection to potential further perceptions and insights. As a result of this constant evolution, terms change in meaning. The term 'planet', for instance, was redefined by the International Astronomical Union in 2006, partly because of the discovery of the Kuiper Belt in 1992.

In order to focus on understanding, we have adopted the term 'unit of understanding', which was originally introduced in sociocognitive terminology theory (Temmerman 2000) in order to deal with the inadequacy of classical concept theory for the conceptual structure of most specialised fields. The unit of understanding pertains to the understanding of a referent studied in a given subject-field. The unit of understanding is communicated via specialised discourse in which it can be expressed in several ways.

The constant development of units of understanding can be explained as the result of several simultaneously active factors: a) the urge for more and better understanding; b) the interaction between different language 
users; c) prototype structure in the understanding of categories which can be seen simultaneously as the result of and as one of the causes of meaning evolution. Cognitive models play an important role in the development of new ideas.

In order to describe units of understanding related to a specific subject area (e.g. biology, informatics, law), sociocognitive terminology studies term behaviour in specialised texts (cf. Section 3). In text linguistics, Collet (2004: 109) defines a term as "a semantically charged linear structure, which names an abstract or concrete reality within a special subject field". In sociocognitive terminology, terms are held to be central in terminological analysis. They can be studied in text corpora, where three types of contexts have to be taken into consideration: the lexical context, the situational context and the cognitive context. The lexical context pertains to the lexical units that precede and follow a particular term. The situational context pertains to the sociocultural and psychological backgrounds of sender and receiver that influences the creation and understanding of a message respectively. The cognitive context concerns the memory stock building up in the course of the assimilation of the meaning of spoken or written discourse. Durieux (1995) argues that these contexts are important for translators to decide on the most suitable translation candidate for a given translation unit in a source language. These contexts are also important for terminographers who need to develop terminological resources in one or several languages.

\section{From theory to practice}

In terminology theory, the perspective is the term. Conscious reflections on the distinction between generalisation and contextualisation in determining the difference between core meaning and various senses of a term are part and parcel of terminological analysis. Theoretical and applied terminology studies increasingly recognize the role of cognition (e.g. Zawada \& Swanepoel 1994, Sager \& Kageura 1995, Weissenhofer 1995, Sager 1998, Temmerman 2000). Methods for the identification, analysis, recording and processing of terms are being developed from a cognitive perspective (e.g. Kerremans et al. 2006). Special attention is given to the role of terms in cognition. Terms are studied from a diachronic perspective in order to get better insight into the role of terminology in a transitional process of understanding (e.g. Dury 2005). Methods of cognitive analysis are being applied in multilingual terminology projects and focus on research into metaphors, the dynamic nature of cognition and the role of language and semantics, terminological variation, etc.

The shift from meaning to understanding (cf. Section 2) has important implications for terminography. Terminography incorporates several

practical tasks, such as the identification and analysis of terms in specialised texts as well as the organisation and description of these units in termi- 
nological resources. Focussing on understanding first of all emphasises the fact that there are different degrees of understanding, depending on the type of user. A technical definition of a given term may be understood by a domain expert but not necessarily by a specialised translator. Consequently, one 'ideal' definition of units of understanding - whereby meaning is defined on the basis of unique and sufficient properties - is often neither possible nor desirable (Temmerman 1997). When terminological resources are developed, it is crucial to start from questions such as 'who are the users and what information do they require?'. Studies of translation processes, for instance, have shown that translators of specialised texts need access to multilingual specialised dictionaries which specify the ways in which terms are structured in a network of intra- and interlingual relations (e.g. Dancette 1994, Temmerman 2003). Intralingual relations specify how terms in a given language and within a given domain are related to one another. Interlingual relations specify how, in a given domain, terms from different languages are semantically related to one another.

With respect to the relations between terms in one language and their translations in other languages, it should be noted that differences on the level of semantics do occur and should therefore be made explicit in multilingual terminological resources. These semantic differences may result from differences in the embodied and situated experience of a given referent by members of cultural groups (cf. Section 2). Many units of understanding are therefore not clear-cut but have prototype structure which should be accounted for in terminological resources, as is, for example, shown in the dictionary by Dancette and Réthoré (2000). The fact that referents may be conceptualised or perceived in different ways, is a possible explanation for both polysemy and synonymy (see e.g. Freixa 2006).

Another aspect that should be accounted for is the dynamic nature of units of understanding (cf. the planet example in the previous section) as a result of the fact that our understanding of reality and of certain phenomena in reality continuously undergoes changes (cf. Section 2). Although this is an issue that we do not wish to address any further as it goes beyond the scope of the present article, it is at least a point that is worth mentioning. Especially in disciplines that are characterised by rapid changes, such as biology and medicine, terminological resources need to reflect the changes that occur on the language level. Many (electronic) terminological resources, however, are usually quite static and are changed or modified only once in a while, mainly because they are managed by one person or by only a few people. We believe that terminological resources can keep up with the changes in disciplines such as biology or medicine much better if their development and maintenance becomes the responsibility of their entire user community. We explored this idea in a project about competencybased occupational profiles, in which a collaborative on-line platform was developed for small and medium-sized enterprises. For further details about this project, we would like to refer to Kerremans et al. (2006). 
The shift from meaning to understanding clearly has implications for terminography, as we have explained up until now. Obviously, some of the issues addressed in the previous paragraphs are also important for (aspiring) translators. Consider, for instance, the fact that a term may have different interpretations, depending on the contexts in which it is used, or that several lexicalisations denoting the same referent may be the result of differences in categorisations and should therefore not be considered as fully equivalent.

In order to become aware of these issues, translators first need to acquire insight into a given domain before they start translating specialised texts. In practical specialised translation courses, aspiring translators are usually asked to read a number of texts in the source and target languages and to extract from these texts terms and translation equivalents which they add to a bilingual terminological database. Although this is a good exercise for students to identify essential units of understanding related to a given subject area, it still needs to be examined whether this level of knowledge suffices to understand the specialised texts and to make the right choices with respect to any translations.

In our view, students have reached a good level of understanding when they not only identify the main units of understanding in a specific domain but are also able to explain how these units are related to one another. For instance, in order to know whether the Dutch term 'exoot' should be translated in English as e.g. 'exotic species', 'alien species', 'alien invasive species', etc., students need to know how these different units of understanding, denoted by the English terms, are related to one another. They also need to be aware that the meaning of terms such as 'invasive species' may vary, depending on the context in which these terms are used. In other words, it is essential that students go a few steps further than just copying terms and definitions from texts and pasting them into a term base (unfortunately, often without a careful reflection about what they have added). They should be critical with respect to the information that they are reading. In order to understand how units of understanding are related to one another, students can be taught how to analyse linguistic contexts in specialised texts which provide essential information to increase their knowledge of one or several units of understanding. It should be noted that it is the trainer of the translation course who decides what texts the students should read in preparation of their translation assignment.

Construing domain knowledge via terminological understanding, is a difficult process for which students should be given efficient support. The CatTerm software developed for guiding students through different knowledge acquisition steps, will be presented in Section 5.

The idea of construing a domain knowledge model, which is the main purpose of the didactic software, is based on termontography, a method set up to construct terminological resources that are supported by ontologies. In Section 4, we briefly present the method and reflect on one important methodological step in particular, that is, the creation of a catego- 
risation framework which defines the scope of each terminological project and allows for the structuring of terms and knowledge-rich contexts (Meyer et al. 1997) extracted from specialised texts.

\section{Construing domain knowledge in termontography}

Termontography combines principles of sociocognitive terminology as well as principles of text-based, application-oriented ontology development (Kerremans et al. 2003). The method was originally set up to support the work of terminographers in projects dealing with the development of multilingual terminological resources (Kerremans et al. 2003).

The application-oriented view in termontography motivates the need for knowledge analysis to precede, firstly, the compilation of a corpus of specialised texts and, secondly, the extraction of terms and other information (such as definitions) from these texts (Kerremans et al. 2003). The aim of the knowledge analysis step is to define the user requirements and scope with respect to the development of an ontologically-underpinned terminological resource or 'termontological resource'.

One of the main tasks in termontography is setting up a categorisation framework. This framework is a model of specialised knowledge which can be created in collaboration with domain experts. It is further refined on the basis of an analysis and understanding of terms in specialised texts. This entire process is now supported by software tools (see e.g. De Baer et al. 2006a).

The categorisation framework, partly shown in Table 1, is composed of units of understanding which we call 'categories' as they are used as a means for classification. Examples of categories in Table 1 are: abdijbier (i.e. 'abbey beer') and alcoholische drank (i.e. 'alcoholic beverage'). As is shown in Table 1, the representation of these categories is not based on formal specifications but relies on lexical items. These lexical items may be single-word terms, complex terms or even longer expressions (cf. Kerremans et al. 2003). Such representations in natural language are easily understood by human users but may cause difficulties when, for example, a term has several possible interpretations (cf. Section 2). For instance, according to the Merriam-Webster on-line dictionary, ${ }^{1}$ the term 'ale' (cf. Table 1) can refer to either an alcoholic beverage or to an English country festival. In the categorisation framework, this problem of ambiguity is solved by linking categories to one another on the basis of different types of relationships. For instance, the English term 'ale' is disambiguated if the two corresponding categories (i.e. one for each interpretation) are linked to different superordinate categories on the basis of a generic-specific relationship, of which we show a possible model in Table 1. 
Table 1: Possible disambiguation of the English term 'ale'

\begin{tabular}{|c|c|c|c|c|}
\hline $\begin{array}{c}\text { English } \\
\text { term }\end{array}$ & is label of & Category $_{1}$ & relates to & Category $_{2}$ \\
\hline \multirow{2}{*}{ ale } & $\rightarrow$ & ale & is subtype of & $\begin{array}{c}\text { alcoholic } \\
\text { beverage }\end{array}$ \\
\cline { 2 - 5 } & $\rightarrow$ & ale & is subtype of & festival \\
\hline
\end{tabular}

Categories may also be linked to one another on the basis of whole-part relationships. For instance, the categories referred to in English as 'hops', 'malt', 'water' and 'yeast' are all part of the category labelled as 'beer'. The relations between these categories are shown in Table 2. Note that in the example, categories and relations are represented by means of terms in English and Dutch.

Table 2: Representation of whole-part relationships

\begin{tabular}{|c|c|c|c|c|c|}
\hline \multicolumn{2}{|c|}{ Category } & \multicolumn{2}{c|}{ relates to } & \multicolumn{2}{c|}{ Category } \\
\hline English & Dutch & English & Dutch & English & Dutch \\
\hline water & water & part of & deel van & beer & bier \\
\hline hop & hop & part of & deel van & beer & bier \\
\hline malt & mout & part of & deel van & beer & bier \\
\hline yeast & gist & part of & deel van & beer & bier \\
\hline
\end{tabular}

It should be noted that apart from generic-specific and whole-part relationships, the categorisation framework also allows us to specify all sorts of associative relationships. In this sense, its structure is much more refined than the structures of thesauri or taxonomies. For a more elaborate discussion on this, we refer to De Baer et al. (2006b and 2008a). From the moment we specify an associative relationship, the category is no longer considered as a means for classification (cf. supra), but becomes a concept. This explains why in Figure 2, the structure carries the (Dutch) name conceptmodel ('concept model').

Apart from adding units of understanding (i.e. categories or concepts), terms and relationships, the categorisation framework also provides the possibility to add all sorts of extra information, such as definitions (cf. Figure 2) or any other type of descriptive information. For instance, in case of a semantic difference between a source language term and its translation equivalent or between denominative variants within the same language (cf. the example of 'Southern blotting' vs 'Southern transfer', which was explained in Section 2), the difference may be explained in a field called 'transfer comment'. A transfer comment is meant to warn users of the termontological resource (e.g. translators) against mistakes in transferring information from one situational or cultural context to another. In the case of describing a possible semantic difference between a source language term and its translation(s), this descriptive field roughly corresponds to the relations internotionelles in the dictionary by Dancette and Réthoré (2000). 
For a more detailed discussion of the categorisation framework in termontography, we refer to Kerremans (2004) and De Baer et al. (2006b, 2008a). In the next section, we will show how the framework is implemented in the CatTerm software tool.

\section{Software support for student translators}

Translators need to go through a number of knowledge acquisition steps if they want to produce high-quality translations of specialised texts (e.g. technical, scientific and legal texts). Relying on a bilingual corpus of specialised texts, they will be introduced to the basics of the relevant domain (e.g. Zanettin 1998, Bowker 1999, Wilkinson 2005), become familiar with the terms that are used in the domain (in both source and target languages) and become aware of the semantic differences between terms, synonyms and translation equivalents, which they can specify in the field of transfer comments (cf. Section 4).

From our own experience in teaching specialised translation courses, we have learned that these knowledge acquisition steps are often highly underestimated by many of the aspiring translators. Since we believe that during translation training more time should be devoted to guiding student translators in a consistent manner through the knowledge acquisition steps, we developed CatTerm.

As a platform-independent interactive computer program, CatTerm guides student translators through four main tasks that we consider necessary for knowledge acquisition:

1) constructing and analysing a bilingual corpus of specialised texts;

2) manually identifying terms in both source and target languages;

3) creating a categorisation framework which is used to classify the terms;

4) describing the terminology in the target language.

The four tasks in the knowledge acquisition process are split up into seven methodological steps. As the purpose of this exercise for students is to come up with a categorisation framework that is based on information in the target language (which is normally the students' native language), they first need to read target language texts from a corpus compiled by the trainer (step 1). It should be noted that students also learn to look for reliable texts on the Internet, similar to those in the basic corpus, and learn to consult alternative sources, such as (multilingual) glossaries, thesauri and specialised translation dictionaries that are relevant for the given domain.

By reading the texts in the corpus, the students will acquire more insight into the domain and become familiar with the terminology in the target language, which they are asked to highlight in each selected text (step 2). As, for example, Zanettin (1998) already pointed out, terms are not only isolated words but can also be wider chunks of language, such as multi- 
word units and recurring word patterns that are found in the corpus. Relying on their understanding of the texts, students start building a categorisation framework (step 3). Afterwards, they assign definitions to terms in the framework (step 4). During this step, they also learn to search for and formulate descriptions for the terminology of the domain.

Next, students select texts from the corpus in the source language (step 5). This corpus also contains the text(s) that they need to translate. They first read a text/texts and identify the terms (step 6). The existing categorisation framework is used as a reference model for structuring the extracted units in the source language (step 7). Additional source language texts may be consulted to complete the categorisation framework. In the end, students are able to generate an ontological bilingual dictionary derived from their own work. In this respect, CatTerm is in fact a kind of terminology management software (e.g. MultiTerm), although the emphasis is much more on the acquisition of domain knowledge in comparison to the compilation of a bilingual terminological resource.

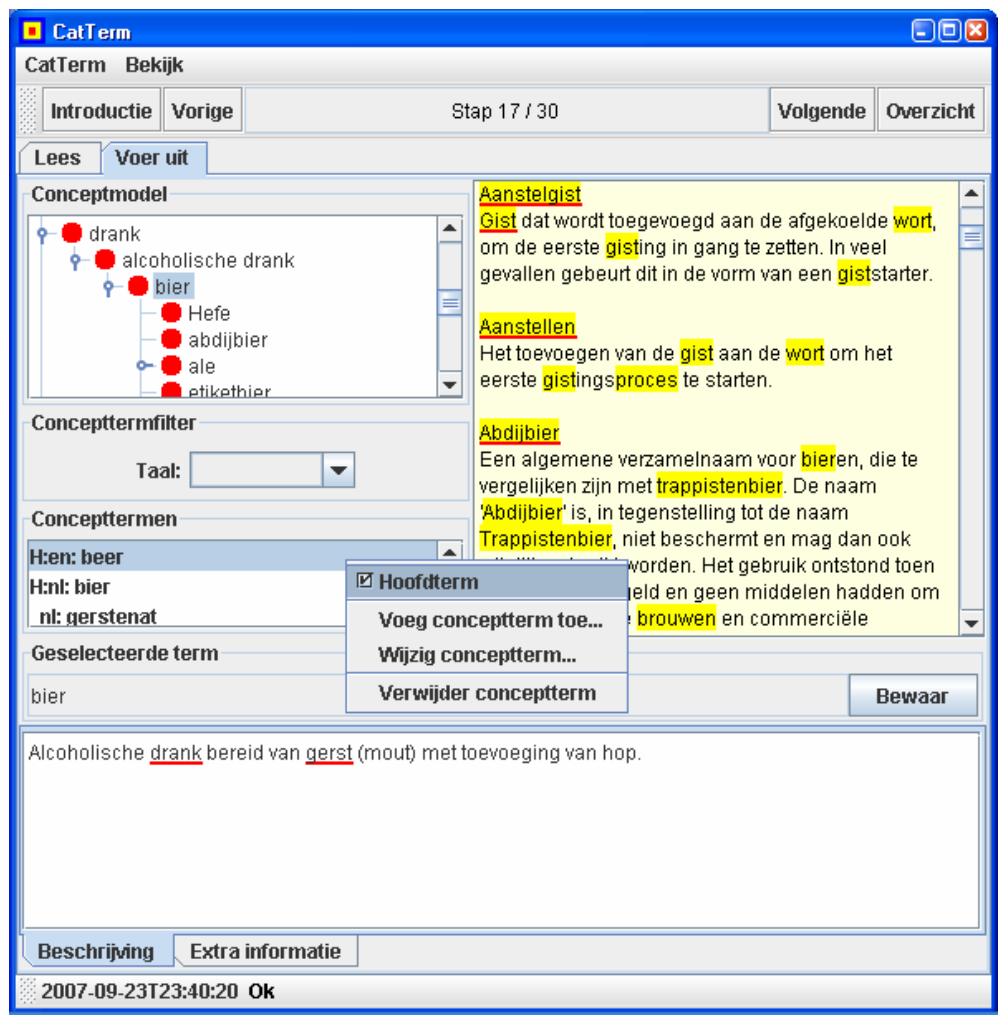

Figure 2: The CatTerm software

Figure 2 is a screenshot of the CatTerm tool. The righthand panel in this screenshot shows the selected text in the target language. In this particular 
example, the text consists of a glossary list of terms that are used to denote units of understanding related to the process of beer brewing. The highlighted words in this example such as 'gist' (yeast), 'abdijbier' (abbey beer) or 'trappistenbier' (Trappist beer) are terms that were selected by the student. These terms are structured in the categorisation framework, which is shown in the left-hand panel. The framework can be viewed in either the source language or target language by specifying the language underneath the categorisation.

By selecting a category in the framework, CatTerm shows the list of terms (including synonyms and translation equivalents) that students have assigned to this category. Terms that are preceded by the ' $\mathrm{H}$ ' label are marked as 'head term'. These terms are used to represent the category in the categorisation framework. Finally, the lower panel in the figure shows that, students can add a definition to a term that has been selected in the term list.

The CatTerm software was tested in the framework of an MA dissertation (Van Poelvoorde 2008). The study showed that the software is effective in the sense that it obliges student translators to be critical about the information that they find in specialised texts and to reflect thoroughly on their understanding of terms and translation equivalents. At the same time, the study listed some disadvantages concerning the usability of the current version and formulated suggestions that may lead to improvements. For instance, our choice to simplify the categorisation process by allowing student translators to only define generic-specific relationships was considered too restrictive. Still, we are not planning to incorporate associative and whole-part relations in CatTerm as this would complicate the classification of terminological information too much. It should be noted, however, that CatTerm allows these relations to be added via text fields. There is also another tool, the Multilingual Categorisation Framework Editor (De Baer et al. 2008a), which allows us to specify generic-specific, whole-part and associative relations between terms, and thus supports the development of more complex categorisation frameworks.

Although CatTerm was developed primarily as a didactic tool to help student translators to acquire domain knowledge, the collected terminological information may also be exported in several formats, to be used during translation tasks. For example, a translation dictionary in HTML format or a translation memory in Character Separated Values format may be generated. Moreover, CatTerm makes use of the Categorisation Framework API (De Baer et al. 2008b) for the construction and storage of terminological information. This ensures that the resulting term base can also be managed with other terminography software tools that support this API. 


\section{Conclusion}

In this article, we have first explained that taking the perspective of understanding instead of the perspective of meaning requires us to add two more dimensions to the traditional semiotic triangle which was visualised in Figure 1 (Section 2).

The first dimension is embodiment, which implies that humans have the faculty to create units of understanding in the mind and to communicate about them. The notion of 'embodiment' emphasises the subjective nature of understanding, derived from an individual's embodied experience of a given referent. This suggests that units of understanding are not equally imprinted in the mind of each individual (Sharifian 2003) and therefore show prototype structure.

The second dimension is situatedness, which implies that units of understanding are culture-dependent as they emerge between members of a cultural group who negotiate and renegotiate them across time and space. Units of understanding are therefore not fixed items but constantly undergo changes. The notion of 'situatedness' emphasises the situational context in which understanding takes place as it determines (only to some degree) the embodied experience of an individual with respect to a given referent.

The prototypical structure of units of understanding should therefore be represented in terminological resources by considering the cognitive and situational contexts over and above the lexical ones in the analysis of terms encountered in specialised texts. We have tried to show how this idea is adopted in the termontography method, a practical working method for developing ontological terminological resources. By discussing CatTerm, we showed how student translators learn to adopt the sociocognitive principles in an attempt to acquire domain insight. By starting from a categorisation framework, which they set up on the basis of an understanding of a bilingual corpus of specialised texts, the students are able to structure and define terms (including synonyms and translation equivalents) and construe domain knowledge.

The work in progress on the impact of the distinction between lexical, situational and cognitive contexts and on the possibilities to apply this distinction in the terminological analysis of a text corpus should provide us with insights into how termontological resources could be adapted when circumstances change, textual corpora are updated or when the domain expands.

\section{Bibliography}

Bowker, Lynne (1999). "Exploring the potential of corpora for raising language awareness in student translators". Language Awareness (8)3/4, 160-173.

Chrisley, Ronald and Tom Ziemke (2002). "Embodiment”. L. Nadel (ed.), Encyclopaedia of cognitive science. London: Palgrave Macmillan, 1102-1108.

Collet, Tanja (2004). "What is a term?". Linguistica Antverpiensia New Series 3, 99-112. 
Dancette, Jeanne (1994). "C. Dollerup \& A. Lindegaard (eds.). Translation and interpreting 2: Insights, aims, visions. Amsterdam: John Benjamins, 113-120.

Dancette, Jeanne and Christophe Réthoré (2000). Dictionnaire analytique de la distribution. Analytical dictionary of retailing. Montréal: Les presses de l'Université de Montréal.

De Baer, Peter, Koen Kerremans and Rita Temmerman (2006a). "Bridging communication gaps between legal experts in multilingual Europe: Discussion of a tool for exploring terminological and legal knowledge resources". E. Corino et al. Proceedings of the XII Euralex international congress. Turin, 813-818.

De Baer, Peter, Koen Kerremans and Rita Temmerman (2006b). "Facilitating ontology (re)use by means of a categorisation framework". In: Meersman, R. et al. On the move to meaningful internet systems 2006. Proceedings of the AWeSOMe workshop. Montpellier, 126-135.

De Baer, Peter, Koen Kerremans and Rita Temmerman (2008a). "A Categorisation Framework Editor for Constructing Ontologically underpinned Terminological Resources”. In: Daille, B. et al. Proceedings of the sixth international conference on language resources and evaluation (LREC), Marrakech [CD-ROM].

De Baer, Peter, Koen Kerremans and Rita Temmerman (2008b). "A Categorisation Framework API for constructing ontology-based lexical resources”. In: Madsen, Bodil Nistrup et al. Proceedings of the eight international conference on Terminology and Knowledge Engineering (TKE), Copenhagen.

Durieux, Christine (1995). "Texte, contexte, hypertexte". Cahier du CIEL (1994-95), 214-228.

Dury, Pascaline (2005). "Terminology and specialised translation: The relevance of the diachronic approach." LSP and Professional Communication 5(1), 31-42.

Freixa, Judit. (2006). "Causes of denominative variation". Terminology 12(1), 51-77.

Fillmore, Charles (1985). "Frames and the semantics of understanding". Quaderni di Semantica 6(2), 222-254.

Fillmore, Charles and Beryl Atkins (1992). "Toward a frame-based lexicon: The semantics of RISK and its neighbors". In: A. Lehrer \& E. Feder Kittay (eds.). Frames, fields and contrasts. Hillsdale, NJ: Lawrence Erlbaum, 75-102.

Geeraerts, Dirk (1986). Woordbetekenis. Een overzicht van de lexicale semantiek. Leuven: ACCO.

Geeraerts, Dirk (1997). Diachronic prototype semantics. A contribution to historical lexicology. New York: Oxford University Press.

Kerremans, Koen (2004). "Categorisation frameworks in termontography". In: Temmerman, Rita and Knops, Eugenia. (eds.). The translation of domain specific languages and multilingual terminology management. Linguistica Antverpiensia New Series 3/2004. Antwerp: Hogeschool Antwerpen, 263-277.

Kerremans, Koen, Rita Temmerman and Jose Tummers (2003). "Representing multilingual and culture-specific knowledge in a VAT regulatory ontology: support from the Termontography approach". Lecture Notes in Computer Science (2889), 662-674.

Kerremans, Koen, Peter De Baer and Rita Temmerman (2006). "Towards A Multilingual Termontological Resource of Competency-based Job Descriptions for SMEs". Proceedings of the International Conference on Terminology, Antwerp.

Meyer, Ingrid, Douglas Skuce, Judy Kavanagh and Laura Davidson (1997). "Integrating linguistic and conceptual analysis in a WWW-based tool for terminography". G. Lessard \& M. Levison (eds.). Proceedings of the joint international conference of the Association for Computers and the Humanities and the Association for Literary \& Linguistic Computing. Ontario: Queen's University. http://www.cs.queensu.ca/achallc97/papers/p021.html

Ogden, Charles Kay and Ivor Armstrong Richards (1923). The meaning of meaning: A study of the influence of language upon thought and of the science of symbolism. London: Kegan Paul, Trench, Trubner.

Poelvoorde, Helmut Van (2008). Opstellen van een categorisatieframework met CatTerm ter voorbereiding van het vertalen van gespecialiseerde (bio)medische teksten over hematologie. Unpublished MA-dissertation. Brussel: Erasmushogeschool Brussel.

Raskin, Victor (1986). "Script-based semantic theory". In: Ellis, D.G. and Donohue, W.A. (eds.). Contemporary issues in language and discourse processes. Hillsdale, NJ: Erlbaum, 23-61.

Rastier, François (1991) Sémantique et recherches cognitives. Paris : PUF.

Sager, Juan C. (1998). "In search of a foundation: Towards a theory of the term". Terminology 5(1), 41-58.

Sager, Juan C. and Kyo Kageura (1995). "Concept classes and conceptual structures: Their role and necessity in terminology". Actes de Langue française et de linguistique 7(8), 191-216.

Sharifian, Farzad (2003). "On cultural conceptualisations". Journal of Cognition and Culture (3), 187-207. 
Temmerman, Rita (1997). "Questioning the univocity ideal. The difference between sociocognitive terminology and traditional terminology," Hermes. Journal of Linguistics 18, 5190.

Temmerman, Rita (2000). Towards new ways of terminology description: The sociocognitive approach. Amsterdam: John Benjamins.

Temmerman, Rita (2003). "Innovative methods in specialised lexicography". Terminology 9(1), 117-135.

Temmerman, Rita (2008). "Sociocultural situatedness of terminology in the life sciences: The history of splicing". F. Roslyn et al. (eds.). Body, language and mind. Vol II. Interrelations between biology, linguistics and culture. Tübingen: Springer.

Vygotsky, Lev (1986). Thought and language (translation of Myshlenie I rech (1934)) Cambridge, MA: MIT Press.

Weissenhofer, Peter (1995). Conceptology in terminology theory, semantics and word formation: A morpho-conceptually based approach to classification as exemplified by the English baseball terminology. Vienna: TERMNET.

Wilkinson, Michael (2005). "Using a specialized corpus to improve translation quality". Translation Journal 9(3). http://www.accurapid.com/journal/33corpus.htm (consulted 09.29.2008).

White, James Boyd (1991) "Our meanings can never be the same: reflections on language and law". Rhetoric Society Quarterly 21(3), 68-77.

Zanettin, Federico (1998). "Bilingual comparable corpora and the training of translators". Meta 43(4): 616-630.

Zawada, Britta E. and Piet Swanepoel (1994). "On the Empirical Inadequacy of Terminological Concept Theories: A case for prototype theory," Terminology 1(2), 253-275.

1 http://www.merriam-webster.com/dictionary/ale 